\title{
Terminología culinaria en De re coquinaria: lengua técnica y coincidencias con el latín vulgar ${ }^{1}$
}

\section{Culinary Terminology in De re coquinaria: Technical Language and Coincidences with Vulgar Latin}

\author{
Amalia LeJAVITZER LAPOUJADE \\ https://orcid.org/0000-0003-0663-1957 \\ Departamento de Comunicación de la Universidad Católica del Uruguay, Uruguay \\ amalia.lejavitzer@ucu.edu.uy
}

RESUMEN: Este artículo examina el uso de la terminología culinaria referida por el recetario De re coquinaria, por tradición atribuido a Apicio. A partir del análisis de términos especializados, se estudia su empleo y su significación técnica, centrado en dos ejes conceptuales: descripción de procedimientos prácticos (verbos) y denominación de realizaciones materiales (sustantivos). Además se muestran recursos de creación del lenguaje técnico latino (como calcos y préstamos lingüísticos del griego y derivación en diminutivo), y se presentan semejanzas de la lengua especializada y el latín vulgar. Se concluye que el lenguaje culinario expresado en el De re coquinaria y el latín vulgar evidencian fuertes coincidencias morfológicas, pero tienen valores expresivos y usos lingüísticos distintos, lo cual pone de manifiesto el carácter polisémico de la terminología culinaria latina.

Palabras Clave: terminología culinaria latina, lengua técnica, latín vulgar, De re coquinaria, Apicio

AвSTRACT: This article examines the use of culinary terminology in the recipe book De re coquinaria, by tradition attributed to Apicius. From the analysis of the specialized terms, its use and its technical significance are studied, focussed on two conceptual axes: description of practical procedures (verbs) and naming of material results (nouns). Also it shows the resources for the creation of technical language (as loan translations of Greek and diminutive derivatives)

\footnotetext{
${ }^{1}$ El punto de partida de este artículo fue una ponencia presentada en el Coloquio Internacional La Traducción de Textos Antiguos (Universidad Nacional Autónoma de México, 5 y 6 de septiembre de 2019). Esta última versión del artículo se ha visto enriquecida con los valiosos aportes realizados por Elbia Di Fabio y Rosario López Grégoris, a quienes expreso mi gratitud. 
as well as its coincidences with Vulgar Latin. It is concluded that the culinary language in the De re coquinaria and the Vulgar Latin have strong morphological coincidences, but with different expressive values and linguistic uses, which highlights the polysemic character of Latin culinary terminology.

KEYwords: Latin Culinary Terminology, Technical Language, Vulgar Latin, De re coquinaria, Apicius

RECIBIDO: 17/03/2020 • ACEPTADO: 07/05/2020 • VERSIÓN FINAL: 17/07/2020

\section{INTRODUCCIÓN}

El De re coquinaria ${ }^{2}$ es una compilación de recetas conformada a lo largo de varios siglos hasta fines del siglo III o comienzos del IV, cuando se fijó por escrito la versión que la tradición manuscrita nos ha conservado. ${ }^{3}$ Sin embargo, se encuentran recetas anteriores, en especial del siglo I de nuestra era. Algunos autores consideran que el núcleo de recetas provenientes de esa época podría estar constituido por los escritos culinarios de la autoría del propio Apicio ${ }^{4}$ o por una reescritura temprana realizada directamente a partir de ellos.

Dicho personaje, conocido y denostado por su enorme fortuna y por sus excesos, vivió bajo el reinado de Tiberio y fue parte de los aristócratas allegados al emperador. No se sabe la fecha precisa de su muerte, pero sí el modo en el que falleció: Apicio se suicidó bebiendo veneno, por temor a caer en la miseria y no poder mantener su tren de vida. ${ }^{5}$ Su nombre trascendió su tiempo, se mantuvo ligado al recetario como su autor e incluso acabó por fusionarse con el título mismo de la obra: Apicii de re coquinaria libri decem o Apicii de re coquinaria.

Este recetario constituye una de las fuentes principales para el conocimiento de la terminología ${ }^{6}$ culinaria latina. Si bien el léxico culinario latino

\footnotetext{
${ }^{2}$ Para los textos latinos del De re coquinaria he seguido la edición de Jacques André (1974), publicada en Les Belles Lettres. En algunos casos he contrastado esa edición con las de Bárbara Pastor Artigues (1987), Giulia Carazzali (1990), Christopher Grocock y Sally Grainger (2006), así como con la traducción de Miguel Ibáñez Artica (1995).

${ }^{3}$ Gómez I Pallarès 1996, p. 176.

${ }^{4}$ Cf. los testimonia citados en la edición de André 1974, pp. xxviii-xxix: 29. Mythogr. 2, 225 : Apicius [...] qui de condituris multa scripsit; 28. Scol. Juvenal, 4, 23: Apicius [...] qui scripsit de iuscellis; 34. Scol. Querolus, p. 22, 17: de condituris multa scripsit.

${ }^{5}$ Cf. Sen., Cons. Helv., 11, 10, 8-9; Mart., 3, 22.

${ }^{6}$ Adoptamos la definición del $D E L$ 2018: terminología es "el conjunto de términos o vocablos propios de determinada profesión, ciencia o materia”. Cabe señalar que terminología también se refiere a la disciplina derivada de la lingüística "que permite identificar el vocabulario de una determinada especialidad de forma sistemática, analizar dicho vocabulario y, si es necesario, crearlo y normalizarlo" (Dubuc 1999, pp. 21-22).
} 
puede rastrearse en manuales de medicina, agronomía y botánica, asimismo en obras de ficción literaria como las sátiras de Horacio y de Juvenal, los epigramas de Marcial, y, por supuesto, el Satiricón de Petronio, el libro de Apicio representa el único corpus en materia culinaria, propiamente dicha, de la antigüedad clásica.

\section{UN LIBRO TÉCNICO}

El De re coquinaria puede situarse entre las obras de literatura técnica latina -designación más precisa que literatura científica-, como sostiene Montero Cartelle. ${ }^{7}$ Fleury expresa que se trata "de textos sobre las técnicas", 8 es decir, escritos especializados cuyo principal rasgo en común es transmitir la aplicación práctica de ciertos conocimientos. Estos textos fundamentalmente se ocupan "de las técnicas [...] necesarias para la vida humana: la alimentación, la construcción y la guerra". ${ }^{9}$

El recetario de Apicio fue un libro para ser empleado en la práctica culinaria. Montero Cartelle señala que el De re coquinaria posiblemente fue usado para complementar la formación de quienes buscaban dominar el oficio, además de los aficionados en la materia. ${ }^{10}$

Por su parte, Alföldi-Rosenbaum considera que este recetario constituye "un manual de trabajo para cocineros y mayordomos, y por lo tanto representa un lenguaje que es técnico y no literario". ${ }^{11}$ Pickstone afirma que De re coquinaria "no es un manual para el novato; [porque] en la mayoría de las recetas se presupone el conocimiento de los elementos de la culinaria". ${ }^{2}$ De hecho, en muchas recetas "las instrucciones son dadas solo para hacer la salsa" 13 que va de acompañamiento, pero ni el procedimiento ${ }^{14}$ para elaborar el plato principal ni los utensilios necesarios son mencionados de manera

${ }^{7}$ Cf. Montero Cartelle 2003, p. 260.

8 Fleury 1990, p. 359: "des textes sur les techniques" (el subrayado es del autor). Todas las traducciones de las citas textuales de los autores contemporáneos son mías, salvo donde se explicita el nombre del traductor.

${ }^{9}$ Fleury 1990, p. 360: “des techniques [...] nécessaires à la vie humaine: la nourriture, la construction et la guerre".

${ }^{10}$ Montero Cartelle 2003, p. 279.

${ }^{11}$ Alföldi-Rosenbaum 1972, p. 8: "a working manual for cooks and housekeepers, and thus it represents a language that is technical and not literary" (las cursivas están en el texto en inglés).

12 Pickstone 1935, p. 168: "is not a handbook for the novice; in most of the recipes a knowledge of the elements of cookery is presupposed".

${ }^{13}$ Pickstone 1935, p. 168: "directions are only given for making the sauce".

14 Norrick señala que los pasos que constituyen el procedimiento para la elaboración del platillo no están "estrictamente ordenados conforme a la secuencia de las operaciones que ellos describen" ("strictly ordered to conform to the order of operations they describe", 1983, p. 174). 
explícita. ${ }^{15}$ Por consiguiente, las recetas apicianas implican tanto el dominio de la técnica culinaria como la comprensión de la terminología propia de dicha técnica.

\section{LENGUA TÉCNICA Y COINCIDENCIAS CON EL LATÍN VULGAR}

De manera genérica y conforme a la etimología, una lengua técnica es el lenguaje propio o característico de una téchne, ${ }^{16}$ en este caso el de la téchne o ars coquinaria. André define lengua técnica como la "lengua específica de un grupo social que se sirve de una técnica o que es especialista en una ciencia". ${ }^{17}$ También llamadas lenguas de especialidad ${ }^{18}$ o lenguas especiales representan "el uso particular que de la lengua común hace un sector social o profesional". ${ }^{19}$ En palabras de García-Hernández, estas lenguas especiales, "usadas principalmente por expertos que atienden a objetos específicos de su profesión, adquieren un carácter técnico". ${ }^{20}$

Callebat, por su parte, considera que los lenguajes técnicos son "el conjunto de las formas y de las maneras de expresión utilizadas para identificar y para describir los procedimientos prácticos y las realizaciones materiales de un arte o de un oficio". ${ }^{21}$ Hago énfasis en procedimientos prácticos y en realizaciones materiales, porque serán los ejes conceptuales de los que me serviré para analizar la terminología culinaria que aparece en De re coquinaria.

Ahora bien, algunos rasgos del latín del De re coquinaria se explican por ser tecnicismos propios de una lengua de especialidad; otros reflejan usos característicos del latín vulgar. ${ }^{22}$ No obstante, los límites que separan el tecnicismo de las palabras cotidianas son difusos.

\footnotetext{
${ }^{15}$ Las recetas, por lo general, omiten la mención de las herramientas necesarias para realizar el procedimiento, no indican con cuál instrumento cortar o dónde moler o en qué clase de recipiente cocinar, cf. Norrick 1983, p. 174.

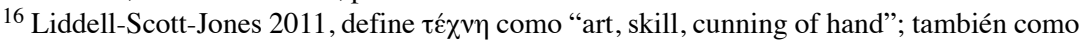
"an art or craft, i.e. a set of rules, system or method of making or doing".

${ }^{17}$ André 1986, p. 8: "langue spécifique d'un groupe social utilisateur d'une technique ou spécialiste d'une science".

${ }^{18}$ Cf. Dubuc 1999, pp. 21 y 45.

${ }^{19}$ García-Hernández 2010, p. 34.

${ }^{20}$ García-Hernández 2010, p. 34.

${ }^{21}$ Callebat 1990, p. 45: "l'ensemble des formes et des modes d'expression utilisés pour identifier et pour décrire les procédés practiques et les réalisations matérielles d'un art ou d'un métier".

${ }^{22}$ Según la conocida definición de József Herman, el latín vulgar es el "conjunto de innovaciones y tendencias evolutivas aparecidas en el uso - sobre todo oral - de las capas latinófonas no influidas o poco influidas por la enseñanza escolar y los modelos literarios" (1997, p. 14). Roger Wright in the foreword to J. Herman, Vulgar Latin apud Grocock y Grainger 2006, p. 95 ,
} 
Por una parte, el recetario de Apicio constituye un documento vinculado a la vida diaria y a un conocimiento que, como el arte culinaria, suele ser transmitido de manera oral. En este sentido, proporciona un vívido testimonio del uso del latín familiar, del sermo cottidianus. ${ }^{23}$ Ejemplo de ello es el empleo del verbo manducare, ${ }^{24}$ el cual es considerado "ejemplo paradigmático de término vulgar". 25

Por la otra, una característica de las lenguas técnicas es denotar realidades cotidianas, por ello se puede decir que son "lenguas vivas", ${ }^{26} \mathrm{y}$, por eso mismo, los textos técnicos, como el De re coquinaria, están fuertemente influidos por el uso vulgar. ${ }^{27}$

Entre los elementos de las lenguas técnicas que también constituyen rasgos distintivos del latín vulgar, destacan dos: el empleo de verbos compuestos con el sentido de la forma simple correspondiente y la profusión de diminutivos. La presencia de verbos compuestos en ambos contextos lingüísticos (el técnico y el vulgar) muestra "que el prefijo ha perdido su fuerza o su significado especializado". ${ }^{28}$ En cambio, la derivación en diminutivo presenta usos bien diferenciados en uno y otro contexto: mientras que en el latín vulgar tiene un claro valor emocional y expresivo, en el discurso técnico se vuelve un recurso de creación de léxico, como ha estudiado López Gregoris. ${ }^{29}$

\section{Algunos hallazgos en De re coquinaria}

A continuación presento algunos ejemplos de términos que describen procedimientos prácticos y otros que identifican realizaciones materiales del arte culinaria, siguiendo las palabras ya citadas de Callebat.

Para referirse a procedimientos es notorio el empleo de verbos compuestos de fundere: effundere, defundere, infundere, perfundere, refundere,

considera que latín vulgar "is just a collective label, available for us to use to refer to all those features of the Latin language that are known to have existed from textual attestations and incontrovertible reconstructions, but that were not recommended by the grammarians". Veikko Väänänen 1995, p. 25 (trad. española Manuel Carrión), considera que el término latín vulgar designa "los diversos fenómenos latinos que no están de acuerdo con las normas clásicas".

${ }^{23}$ La expresión la hizo célebre Cicerón, en Epistulale ad Familiares, 1, 1, 2: in sermone quotidiano; también, 9, 21, 1: quotidianis verbis.

${ }^{24}$ Apic., II, 1, 4; III, 10, 4; IV, 2, 12; VI, 2, 2; VII, 2 , 2.

${ }^{25}$ Montero Cartelle 2003, p. 267. También cf. Väänänen 1995, pp. 50-51, trad. española Manuel Carrión; Herman 1997, p. 118, trad. española María del Carmen Arias Abellán.

${ }^{26}$ Callebat 1990, p. 56: "langages vivants".

${ }^{27}$ Herman 1997, p. 15, trad. española María del Carmen Arias Abellán.

${ }^{28}$ Milham 1959, p. 69: "that the prefix has lost its force or specialized meaning".

${ }^{29}$ López Gregoris 2005, p. 77. Cf. también p. 79. 
profundere, suffundere, superfundere. ${ }^{30}$ En el recetario, todas estas formas aluden a la acción de verter un líquido sobre un sólido, bien sea para ligar los ingredientes de una mezcla o para culminar la preparación de un platillo, bañándolo con una salsa o con miel antes de servirlo. En cualquiera de los casos se ha perdido el significado propio de la partícula preverbal, como se advierte en las fórmulas finales: ius perfundis et inferes, ${ }^{31}$ "bañas con la salsa y servirás", 32 o mel superfundis et inferes, ${ }^{33}$ "bañas con miel y servirás", también oenogaro profundis, insuper piper aspargis et inferes, 34 "bañas con enógaro, ${ }^{35}$ esparces pimienta por encima y servirás". Más aún, la pérdida de sentido de los prefijos verbales incluso da como resultado la formación de verbos supercompuestos ${ }^{36}$ mediante la adición de un segundo prefijo a una forma que ya es compuesta, ${ }^{37}$ como superinfundere. ${ }^{38}$

Otro verbo compuesto empleado con igual significación que su forma simple es contemperare. Este aparece una única vez en el recetario con el mismo significado técnico que temperare, ${ }^{39}$ con el sentido de mezclar ${ }^{40} \mathrm{o}$ con el más específico de ablandar o suavizar: ${ }^{41}$ mittes in mortario piperis

${ }^{30}$ Cf. Marchese 1987, p. 17; Grocock y Grainger 2006, p. 87.

${ }^{31}$ Apic., VI, 8; también VI, 9, 9: iure perfundis et inferes.

${ }^{32}$ Todas las traducciones del latín y del griego al español son de mi autoría.

${ }^{33}$ Apic., VII, 13, 3; VII, 13, 8: melle perfundis, piper aspargis et inferes; VII, 13, 6: perfundis mel, piper aspergis et inferes.

${ }^{34}$ Apic., IV, $5,1$.

${ }^{35}$ El término latino oenogarum es un calco del griego oivó $\gamma \propto \varrho o v$, formado de oîvos (vino)

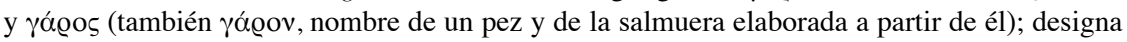
al líquido hecho de garo mezclado con vino y especias, empleado como aderezo o salsa para condimentar diversos platos. Apicio (IV, 5, 3) proporciona una receta para elaborarlo: "Así harás el enógaro: molerás pimienta, levístico, viertes vino y garo, mezclarás con vino paso, echas un poco de aceite al cazo y harás que hierva. Cuando haya hervido, ligas con almidón [...]" (Oenogarum sic facies: teres piper, ligusticum, suffundis uinum et liquamen, passo temperabis, olei modicum mittis in caccabum et facies ut ferueat. Cum ferbuerit, amulo obligas [...]). El garo y sus derivados también fueron usados como tónicos medicinales por las abundantes propiedades digestivas que se les atribuyó (cf. Apic., I, 31; 34). Se trató este tema en Lejavitzer 2000, pp. 115-128, y Lejavitzer 2006, pp. 123-139.

${ }^{36}$ Cf. Väänänen 1995, p. 159, trad. española Manuel Carrión.

${ }^{37}$ Cf. Milham 1959, p. 69.

${ }^{38}$ Apic., VIII, 7, 14: ova XV superinfunduntur.

${ }^{39}$ Temperare aparece 79 veces en De re coquinaria y diez veces en los Excerpta. Además de los matices de mezclar, suavizar, ablandar, pareciera tener uno semejante al de condire, es decir, condimentar o sazonar (Apic., VIII, 6, 11: omnia teres, suffundes liquamen, temperabis ex sale, "todo esto molerás, verterás garo, sazonarás con sal"). También podría tener el sentido técnico de los verbos corregir o rectificar el sabor de un platillo, semejante a la fórmula moderna "prueba y corrige, según sea necesario", "taste and adjust accordingly" (Grocock y Grainger 2006, p. 88), por ejemplo, en Apic., VI, 9, 1, y IX, 84.

40 Apic., III, 4, 2: teres piper, cuminum, rutam, suffundes acetum, liquamen, temperabis in caccabum, "molerás pimienta, comino, ruda, verterás vinagre, garo, mezclarás en el cazo".

${ }^{41}$ Apic., I, 12, 9: senapi tempera melle, "ablanda la mostaza con miel”; también IV, 3, 2: et sic temperas ut tenerum sit, "y así ablandas, para que esté tierno". 
scripulos VIII, suffundes liquamen, fricabis. Adicies sorba, in se contemperabis [...], "echarás al mortero 8 escrúpulos ${ }^{42}$ de pimienta, verterás garo, mezclarás. Añadirás las serbas y mezclarás entre sî". 43

Asimismo, el verbo subassare, compuesto de assare, generalmente traducido por asar un alimento a las brasas o a la parrilla y empleado como sinónimo de la forma simple, constituye otro caso donde la partícula preverbal ha perdido su sentido tradicional. En De re coquinaria, subassare presenta un significado especializado que pone de manifiesto la "compleja polisemia" de la partícula $s u b$, estudiada en profundidad por García-Hernández, ${ }^{44}$ pues parece indicar una ligera cocción inmediatamente antes o después de otro cocimiento. ${ }^{45}$

Cuando la cocción se realiza previamente, tal vez se asemeje al actual tecnicismo culinario sellar o bresear (scellager o braiser, en francés o braise, en inglés) referido a la acción de "dorar carne rápidamente", ${ }^{46}$ para cerrarla herméticamente, a fin de impedir que pierda sus jugos durante el cocido prolongado al que luego será sometida ${ }^{47}$ Así pareciera indicarlo la receta apiciana para preparar un plato de pescado: accipies pisces, curatos subassabis, postea eos in pulpas carpes, "tomarás los peces, una vez limpios, los asarás ligeramente, después los cortarás en trozos", para posteriormente cocinarlos, hasta hacerlos hervir en la salsa de ostras que se ha preparado con enógaro: "untarás la fuente y colocarás el pescado trozado, descrito arriba, en la salsa de ostras. Harás que hierva (patinam perunges et in pulpam supra scriptam mittes et condituram de ostreis. Facies ut ferueat). ${ }^{48}$

Además, subassare figura en las fórmulas de clausura de las recetas para indicar que a veces la carne, ya cocida, se tostaba ligeramente, ${ }^{49}$ como toque final de la preparación antes de servirla: "bañas la carne hervida y después dorada con sal, rocías pimienta y servirás", carnem elixam sale subassatam

${ }^{42}$ Un escrúpulo (scripulum o scrupulum) corresponde a $1 / 24$ de una onza (uncia $=27,25 \mathrm{~g}$ ), es decir, 1,1 g. El DLE 2018 (s. v. escrúpulo) consigna que esta antigua medida de peso, utilizada en farmacia, era equivalente a 24 granos.

${ }^{43}$ Apic., IV, 2, 33.

${ }^{44}$ García-Hernández 1978,p. 41.

45 Cf. Guillén 1981, pp. 216-217, señala que metafóricamente sub indica proximidad de tiempo, esto es, poco antes o poco después.

${ }^{46}$ Tame de Patjane 1998, s. v. braise, p. 97.

${ }^{47}$ El Larousse gastronomique en español bajo la voz bresear (calcada del francés braiser; no incluida en el $D L E$ ) explica que "el término bresear se emplea también para la cocción de ciertos pescados de carne firme [...], que se parece más bien a un escalfado o pochado"; más adelante, añade "se comienza friendo en un cuerpo graso [...] hasta que adquiera un bonito color [...]. Este dorado concentra los jugos en la pieza breseada" (2004, p. 150).

48 Apic., IV, 2, 31.

${ }^{49}$ Cf. Grocock y Grainger 2006, p. 92: "The word thus has the added meaning of 'finish' the cooking by roasting over charcoal". 
perfundis, piper aspargis et inferes..$^{50}$ Entonces, subassare tendría el significado propio del tecnicismo moderno dorar, es decir, tostar ligeramente un alimento para que tome un color dorado..$^{51}$

De las diez veces que subassare aparece en el recetario, en ninguna de ellas sub- presenta el valor tradicional de bajo o debajo, sino el de un poco o ligeramente, o inmediatamente después (este último matiz es el que se advierte en las fórmulas de clausura citadas). García-Hernández ha destacado "la función posicional no-vertical" de sub- (en oposición a prae-), "que aporta al término modificado el contenido espacio-temporal", con el sentido de "a continuación", "después", pero con idea de inmediatez entre la anterioridad y la posterioridad, pues no hay una posición intermedia entre ambas acciones.$^{52}$ Esto es claro en la siguiente receta para preparar la carne de ciervo, la cual "hervirás y a continuación dorarás" (cervum elixabis et subassabis); ${ }^{53}$ para finalizar la bañarás con la salsa hecha de miel, vinagre, garo y especias.

Según García-Hernández, de la función posicional deriva la "función cuantitativa" de sub- con los significados de "un poco", "menos", "algo" (también opuesta a prae- en sus sentidos de "mucho" o "más"). ${ }^{54}$ Este matiz cuantitativo está presente en subassare, según hemos señalado, y se conserva hasta nuestros días en dos verbos de la jerga culinaria: el propio soasar, es decir, "medio asar o asar ligeramente", ${ }^{55}$ y el más frecuente sofreír, cuyo significado es "freír un poco o ligeramente algo" ${ }^{56}$

Respecto a las realizaciones materiales propias del arte culinaria, en el De re coquinaria se encuentran ejemplos de calco y préstamo lingüístico a partir del griego. Así, surgen voces para nombrar utensilios de cocina

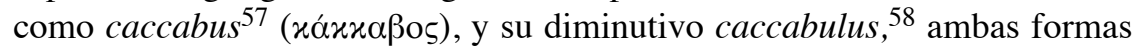
empleadas como sinónimos para referirse a un cazo o cazuela. Thermos-

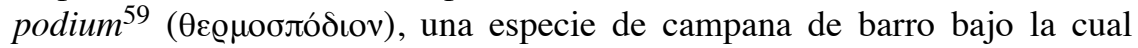
se colocan los alimentos (en especial, panes), que se recubre de brasas o

${ }^{50}$ Apic., VII, 5, 3. En VIII, 2, 3, se lee ceruum elixabis et subassabis, pero nada nos asegura que las acciones efectivamente se realizaran en ese orden.

${ }^{51}$ Cf. Delgado 1997, s. v. dorar.

${ }^{52}$ Cf. García-Hernández 1978, p. 45.

${ }^{53}$ Apic., VIII, 2, 3: cervum elixabis et subassabis. Teres piper, ligusticum, careum, apii semen, suffundes mel, acetum, liquamen, oleum. Calefactum amulo obligas et carnem perfundes.

${ }^{54}$ Cf. García-Hernández 1978, p. 46.

55 DLE 2018, s. v. soasar.

${ }^{56}$ DLE 2018, s. v. sofreír.

${ }^{57}$ Apic., passim. Caccabus, mencionado en al menos 75 ocasiones, es el término preferido para referirse a una vasija honda (olla solo aparece 9 veces), y sobre todo se encuentra en las recetas de verduras hervidas (III, 2, 5; 4, 2; 20,5) y en las preparaciones de guisos y potajes (IV, 2,$5 ; 14 ; 15 ; 31 ;$ IV, 3,$1 ; 2 ; 4-7 ;$ IV, 4,1 y 2 .

${ }^{58}$ Apic., IV, 1, 1 y $2 ;$ IV, 4, 1; 5, 1; VI, 8, 13; VIII, 8, 4.

${ }^{59}$ Apic., IV, 2, 4; 8; 9; 33; IX, 8, 3. 
cenizas para realizar la cocción. Similar en forma y función es el clibanus $\left(x \lambda \hat{i} \beta \alpha v_{0}\right),{ }^{60}$ cierta clase de horno, a veces portátil, semicircular o acampanado, hecho en metal o terracota con una doble pared, que permite que el calor se distribuya en el espacio que queda entre ambas.

También tomado del griego, calix $(x u ́ \lambda \xi \xi)$ designa un tipo de copa; en Apicio, aparece una sola vez, en un contexto de medidas de cantidad: liquaminis optimi calicem. ${ }^{61}$ No es adecuado traducirlo por "copa", puesto que esta voz alude al servicio del vino para su degustación, pero aquí no se refiere ni a dicha bebida ni a su consumo, por lo que parece más apropiado traducirlo por "una medida" o en todo caso por "un vaso" (del mejor garo). Otro recipiente pequeño utilizado como medida es el cyathum, nombre calcado del griego xúaӨos, que designa un vasito con un asa usado a modo de cucharón para trasvasar el vino de la cratera a la copa. A diferencia del calix, el ciato fue una unidad de capacidad, equivalente a la doceava parte de un sextario $(0,045 \mathrm{~L})$, pero en De re coquinaria resulta difícil de determinar, si cyathum está empleado en sentido amplio o como medida precisa. ${ }^{62}$

Caso semejante al anterior es el de acetabulum, "vinagrera" o vasija para contener vinagre. Stricto sensu, el término refiere a una medida de cantidad equivalente a una cuarta parte de una hemina (del griego i $\mu$ ívo), esto es, medio sextario (poco más de 250 mililitros) ${ }^{63}$ Sin embargo, con frecuencia es empleado, lato sensu, con el significado de "pequeño recipiente", a modo de nuestra "taza": ad unum liquaminis acetabulum aquae septem mittes, "echarás una taza [sc. una medida] de garo a siete de agua" ${ }^{64}$ El término resulta tan genérico e impreciso que el autor se ve en la necesidad de acotar el tamaño, en un contexto lingüístico que pone en evidencia, una vez más, una expresión de clara cotidianidad: oleum acetabulum maiorem, [...] liquaminis acetabulum minorem, aceti acetabulum perquam minorem, "una taza muy grande de aceite, $[\ldots]$ una taza pequeña de garo, una taza más pequeña de vinagre". 65

En De re coquinaria también abundan los diminutivos, llamados de "medida" o de "cantidad que cabe en", 66 como scripulum, pugillum, pusillum (cuyas respectivas formas positivas son scrupus, pugnus, pusio), literalmente "piedrita", "puñito", "chiquito" (o "poquito").

Según señala López Gregoris, en las obras técnicas, el empleo de diminutivos sin valor afectivo ni connotativo para referirse a utensilios constituye

\footnotetext{
${ }^{60}$ Apic., VII, 5, 5; VII, 8; VIII, 6, 6; VIII, 9.

${ }^{61}$ Apic., II, $2,4$.

${ }^{62}$ Cf. Apic., IV, 2, 4; 5; 8; 9; 31; 33; 36; VI, 8, 11; VII, 4, 4.

${ }^{63}$ Cf. Apic., VII, 5, 4; VIII, 6, 5.

${ }^{64}$ Apic., II, $2,5$.

${ }^{65}$ Apic., VI, 8, 3.

${ }^{66}$ Cf. López Gregoris 2016, p. 184, y 2005, p. 91.
} 
un recurso de creación léxica extendido y bien documentado. ${ }^{67}$ En estos casos no hay cambio de significado, pues el diminutivo "designa el mismo objeto de la base léxica en tamaño menor". 68 Tanto es así que es usado como sinónimo y ambas formas (la disminuida y la positiva) resultan términos intercambiables sin hacer distinción entre uno y otro objeto.

Además del citado caccabulus ("cazuela"), ${ }^{69}$ como sinónimo del sustantivo simple caccabus ("cazo") está patella (derivado de la forma positiva patina o patena) para referirse al recipiente de bronce o de barro de bordes bajos y extendido, utilizado tanto para cocinar como para llevar a la mesa. Patella y patina (patena) aparecen usados en clara sinonimia en las recetas del capítulo segundo (Patinae piscium, olerum, pomorum) del libro cuarto donde se reúnen las recetas para elaborar esa comida. ${ }^{70}$ En este caso, el recipiente ha dado nombre a la preparación, dulce o salada, que contiene. En el recetario de Apicio encontramos patinae de peras, de albaricoques o de membrillos, y también de espárragos, de calabacitas, de pescado, de anchoas. Al igual que en español "paella", remite tanto al alimento como al recipiente ${ }^{71}$ donde se prepara y después se sirve. Más aún, la voz paella (valenciana para unos, catalana para otros) deriva de la patella latina. ${ }^{72} \mathrm{Y}$ acaso la constitución de ambos platos guarda ciertas semejanzas - excepto por el arroz y el azafrán en la primera y la adición de huevos en la segunda-; se parecen en la forma del recipiente, en la modalidad de cocción y en la variedad de ingredientes que reciben: pescado, mariscos, pollo, incluso embutidos, vegetales diversos como alcachofas, espárragos, cebollas, calabacitas (zucchini).

Otra comida que remite tanto al ingrediente principal de la preparación como al recipiente que la contiene es conchicla. Este es el diminutivo contracto de conchis, proveniente de xó $\chi \chi 0 \varsigma$, sustantivo que en griego se refiere a algo con forma semejante a la del caparazón bivalvo de un molusco. No debe sorprender que las seis recetas reunidas en el capítulo cuarto del libro V del De re coquinaria, bajo el título Conchicla, no estén dedicadas a

${ }^{67}$ Cf. López Gregoris 2016, pp. 182-183.

${ }^{68}$ Cf. López Gregoris 2016, p. 180.

${ }^{69}$ Apic., IV, 4, 1; IV, 5, 1; VI, 8, 13; VIII, 8, 4.

${ }^{70}$ Cf. Apic., IV, 2, 14: Patinam Apicianam sic facies. [...] et in patella alternis de trulla refundes. [...] Patellam aeneam qualem debes habere infra ostenditur. Id., 15: Patina cotidiana. [...] Accipias patellam aeneam [...].

${ }^{71} \mathrm{Si}$ bien la voz paellera quizá sea más frecuente para nombrar el recipiente, cabe señalar que el DLE bajo la voz paella proporciona dos significados: "plato de arroz seco, con carne, pescado, mariscos, legumbres, etc., característico de la región valenciana, en España" y "sartén en que se hace la paella"; mientras que en la entrada paellero,-ra el significado de "recipiente metálico a modo de sartén, de poco fondo y con asas, que sirve para hacer paella" figura en la quinta y última acepción del término.

72 Cf. Corominas 1973, s. v. paila. 
los moluscos (estos se encuentran en el libro décimo del recetario), sino a los potajes hechos con frijoles, guisantes y habas. La morfología de las leguminosas, por la vaina que las recubre, recuerda de modo figurado a las conchas, pero el título del capítulo además se explica porque - al igual que patina y patella - el alimento toma, de manera metonímica, el nombre del recipiente (de barro rojizo, característico de la región de Cumas) en el que se cocina y se sirve. ${ }^{73}$

Igualmente un enser culinario cuya morfología corresponde a un diminutivo es craticula ${ }^{74}$ que puede traducirse por "parrilla" o "rejilla". En Apicio, asar en craticula es una alternativa de cocción al horneado: sale aspargis et in furnum mittis vel in craticulam, "espolvoreas con sal y llevas al horno o a la parrilla". 75

Entre las realizaciones materiales, sin ser utensilios, sino nombres de preparaciones o productos derivados se cuenta ofella (diminutivo de la forma positiva offa, que no figura en el recetario), literalmente, mordidita, bocadito o trocito. Sin embargo, como término culinario, no resulta tan claro su significado, pues no sabemos con exactitud en qué consistía el alimento de ese nombre: podríamos pensar en una especie de albóndigas pequeñas o en trocitos (cubitos o rollitos) de carnes varias que, a modo de pinchos o brochetas, se servían como entrada para tomarla con los dedos ${ }^{76}$ López Gregoris señala que el diminutivo se emplea para expresar la mezcla, es decir, "el resultado de la combinación de varios ingredientes, cuya mezcla origina un producto final altamente transformado", 77 y menciona como ejemplos del español morcilla, picadillo o tortilla, entre otros. En México, a cierta clase de pan blanco se le dice bolillo. Quizá con algún parecido a este

${ }^{73}$ Para referirse a la vasija, en Apic., V, 4, 5, aparece el hápax conciclarem.

${ }^{74}$ Cf. Mart., 14, 221.

${ }^{75}$ Cf. Apic., VII, 2, 1; también VIII, 8.

${ }^{76} \mathrm{El}$ tecnicismo ofellae es traducido de muy diversas maneras: André lo vierte en francés por ragoûts, que en español sería ragús, esto es, el nombre genérico de un platillo hecho a base de carne de ternera, res, ave o pescado, cortada en trozos regulares, normalmente en forma de cubitos o de tiras pequeñas (Delgado 1997, s. v. ragú). En catalán, Gómez I Pallarès (1990) se sirve del también galicismo fricassé: "guiso de carne cortada en pequeños trozos" (Delgado 1997, s. v.fricasé); en español, encontramos estofados en Ibáñez Artica y carnes en Pastor Artigues, y, en italiano, Carazzali traduce por braciole, "costilla" o "chuleta". Por su parte, Grocock y Grainger mantienen el término en latín y lo explican en "A glossary to Apicius", al final de su edición (2006, p. 354): "ofellae can mean lumps of meat, generally small enough to be eaten with the fingers" ("ofellae puede significar cubitos de carne, en general lo suficientemente pequeños para comerse con los dedos"), y más adelante lo definen como a little snack ("pequeño aperitivo", en México se dice "botanita"). Sin embargo, a la luz de las mismas recetas de Apicio, VII, 4, 1 y 2, al parecer los trozos de carne se ensartan en un pincho, lo cual nos lleva a pensar que tal vez las ofellae romanas fueran unas brochetas servidas como aperitivo, quizá semejantes a los actuales "pinchos" españoles. Cf. Varr., L. L., 5, 22, 110: offula ab offa, minima suere ("offula proviene de offa, pequeño trozo de carne de cerdo").

${ }^{77}$ López Gregoris 2016, p. 185. 
último podría ser el término latino buccella, ${ }^{78}$ bocadito (diminutivo de buccea, bocado), ya que en las recetas apicianas siempre está referido a trozos de pan. Tal vez buccella fuera la denominación de algunos panes pequeños para bocadillos, por usar otro "diminutivo culinario".79

Hasta aquí estos ejemplos, cuya finalidad es meramente ilustrativa de la terminología culinaria en el De re coquinaria y de ninguna manera pretenden conformar un repertorio exhaustivo.

\section{A MODO DE CONCLUSIÓN}

El lenguaje de especialidad que muestra el De re coquinaria no debería considerarse inseparable del latín vulgar, pues, más allá de las coincidencias morfológicas que presentan, uno y otro constituyen ámbitos lingüísticos bien diferenciados con funciones discursivas distintas.

De hecho, en la abundancia de diminutivos que registra el recetario es necesario advertir, además de una influencia de la oralidad y del habla cotidiana, "un medio de creación propio de la lengua latina para el lenguaje técnico". ${ }^{80}$ En el empleo de verbos compuestos como sinónimos de sus formas simples correspondientes, hay que ver no solo la pérdida del valor tradicional de las partículas preverbiales - lo mismo sucede en el latín vulgar-, sino también una especificidad de la lengua técnica que encuentra en la alternancia de sinónimos para expresar una misma idea un rasgo propio. Otras veces, el significado especializado de la forma verbal compuesta nada más resultaría evidente para el experto y para el práctico en el oficio, pero no para el lego (como en subassare).

La polisemia de las voces técnicas en parte puede explicarse por el hecho de que, en palabras de García-Hernández, "el léxico de las lenguas especiales se nutre en principio de la lengua común", ${ }^{81}$ es decir, el sentido genérico que tiene una palabra en la lengua común se diversifica en distintos ámbitos donde adquiere un uso técnico especializado. ${ }^{82}$ No obstante, este proceso puede ser de ida y vuelta, pues a veces el tecnicismo regresa a la lengua común; entonces, pierde su sentido técnico, ${ }^{83}$ y "la palabra se banaliza por una suerte de detecnificación". 84

La complejidad está en que la adaptación del significado hacia otros nuevos no suprime el contenido original del vocablo. Como expresa André, "el

\footnotetext{
${ }^{78}$ Apic., VII, 6, 4; VII, 9, 2 y 3; VII, 11, 3; VIII, 6, 1.

${ }^{79}$ López Gregoris 2016, p. 185.

${ }^{80}$ López Gregoris 2005, p. 77. Cf. también p. 79.

${ }^{81}$ García-Hernández 2010,p. 35.

82 Cf. García-Hernández 2010, p. 30.

${ }^{83}$ Cf. García-Hernández 2010, p. 35.

${ }^{84}$ André 1986, p. 11.
} 
neologismo semántico [...] tiene la ventaja de proporcionar un término integrado en el sistema de la lengua sin que la polisemia sea un problema". 85 Por ello, si se considera su carácter polisémico y ambiguo, el lenguaje técnico se aproxima a lo que se conoce como argot, cuyo origen está determinado por el deseo de mantener de manera críptica la comunicación entre los individuos de un mismo grupo. Callebat incluso va más allá, cuando afirma que los lenguajes técnicos son "lenguajes de iniciados". 86

Aunque las lenguas especiales pretenden "una mayor precisión expresiva que la lengua común", 87 esto se cumple únicamente cuando el significado del tecnicismo puede ser interpretado gracias al contexto ${ }^{88}$ y al conocimiento compartido por los versados en la $\tau \dot{\chi} \chi v \eta$. En este sentido, la terminología culinaria latina y la lengua técnica de Apicio en gran medida solo pueden explicarse a la luz de sí mismas, por el hecho de que, del extenso conjunto de obras en materia culinaria de la antigüedad griega y romana, nada más ha sobrevivido el De re coquinaria. ${ }^{89}$ Quizá este sea el sentido de las palabras de Apicio, cuando afirma ad mensam nemo agnoscet quid manducet, "a la mesa, nadie sabrá lo que come". ${ }^{90}$

\section{BIBLIOGRAFÍA}

\section{Fuentes antiguas}

ApICI, L'Art de la cuina, text rev. \& trad. Joan Gómez I Pallarès, Barcelona, Fundació Bernat Metge (Escriptors llatins, 264), 1990, texto enfr., 3 ind.

APIcio, Cocina romana, ed. y trad. Bárbara Pastor Artigues, Madrid, Coloquio, 1987.

ApIcıo, L'arte culinaria: manuale di gastronomia classica, trad. Giulia Carazzali, pres. Francesco Maspero, Milano, Tascabili Bompiani, 1990.

Apicius. A critical edition with an introduction and an English translation of the Latin recipe text Apicius, eds. Christopher Grocock and Sally Grainger, ills. Dan Shandrake, Totnes, Prospect Books, 2006.

ApIcius, De re coquinaria, text. ét., trad. et comm. Jacques André, Paris, Les Belles Lettres (Collection des Universités de France, Guillaume Budé), 1974.

85 André 1986, p. 10: "Le néologisme sémantique [...] a l'avantage de fournir un terme intégré au système de la langue sans que la polysémie soit une gêne".

${ }^{86}$ Callebat 1990, p. 56: "langages d'initiés".

87 García-Hernández 2010, p. 34.

${ }^{88}$ Cf. André 1986, p. 10.

${ }^{89}$ Baldwin 1987, p. 248: "De re coquinaria is the only extant ancient specimen of the genre".

${ }^{90}$ Apic., IV, 2, 12. 
ApIcius, De re coquinaria. Gastronomía en la antigua Roma Imperial, com. y trad. Miguel Ibáñez Artica, San Sebastián, R \& B Ediciones, 1995.

Cicero, M. Tulli, Epistulae ad Familiares, ed. Louis Claude Purser, Oxonii, Clarendon, 1901-1903, http://www.perseus.tufts.edu/hopper/text?doc=Perseus\%3atext \%3a1999.02.0009 (17/07/2020).

Martialis, Epigrammaton liber, text. ét. et trad. H. J. Izaac, Paris, Les Belles Lettres (Collection des Universités de France, Guillaume Budé), 1930-1933, 3 vols.

Seneca, De Consolatione ad Helviam, Moral Essays Vol. 2, ed. John William Basore, London, Heinemann, 1932, http://www.perseus.tufts.edu/hopper/text?doc= Perseus:text:2007.01.0017 (17/07/2020).

VARrón, De lingua Latina, ed. bilingüe, intr., trad. y nts. Manuel-Antonio Marcos Casquero, Barcelona, Anthropos-Ministerio de Educación y Ciencia (Textos y documentos, Clásicos del Pensamiento y de las Ciencias, 6), 1990.

\section{Fuentes modernas}

Alföldi-Rosenbaum, Elisabeth, "Apicius, De re coquinaria and the Vita Heliogabali”, Bonner Historia Augusta Colloquium 1970, Bonn, J. Straub, 1972, pp. 5-10. ANDRÉ, Jacques, "Sur la constitution des langues techniques en latin", Etudes de Lettres, 1, 1986, pp. 5-18.

Baldwin, Barry, An Anthology of Later Latin Literature, Amsterdam, J. C. Gieben, 1987, pp. 248-251.

CAllebat, Louis, "Langages techniques et langue commune", Latin vulgaire-latin tardif II. Actes du II ème Colloque international sur le latin vulgaire et tardif, Bologne, 29 août-2 septembre 1988, Tübingen, M. Niemeyer, 1990, pp. 44-56.

Corominas, Joan, Breve diccionario etimológico de la lengua castellana, Madrid, Gredos, 1973.

Delgado, Carlos, Diccionario de gastronomía, Barcelona, Altaya, 1997.

Dubuc, Robert, Manual de terminología, trad. Ileana Cabrera, Santiago de Chile, RIL Editores, 1999.

Fleury, Philippe, "Les textes techniques de 1'Antiquité. Sources, études et perspectives", Euphrosyne, 18, 1990, pp. 359-394.

García-Hernández, Benjamín, "Desarrollo polisémico del preverbio sub- y su posición en el sistema preverbial", Helmantica: Revista de filología clásica y hebrea, 29/88, 1978, pp. 41-50.

GarcíA-Hernández, Benjamín, 'La polisemia de ius, iuris ('derecho', 'aderezo') y la idea genuina ('unión') del Derecho Romano", Revista de Estudios Latinos, 10, 2010, pp. 29-47.

Gómez I PAllarès, Joan, "La transmisión textual del De re coquinaria de Apicio: último estado de la cuestión", Fortunatae, 8, 1996, pp. 173-189.

Guillén, José, Gramática Latina. Histórico-teórico-práctica, Salamanca, Sígueme, 1981.

Herman, József, El latín vulgar, trad. española María del Carmen Arias Abellán, Barcelona, Ariel, 1997.

Larousse gastronomique en español, Barcelona, Larousse, 2004. 
LEJAVITZER, Amalia, "Garum paradoxum: misterio y maravilla de la cocina romana", Nova Tellus, 18/2, 2000, pp. 115-128.

LejavitZer, Amalia, "Algunas recetas médicas en el De re coquinaria de Apicio", Nova Tellus, 24/1, 2006, pp. 123-139.

Liddell, Henry George, Robert ScotT and Henry Stuart Jones, The Online Liddell-Scott-Jones Greek-English Lexicon, 2011, http://stephanus.tlg.uci.edu/ 1sj/\#eid=106629 (17/07/2020).

López Gregoris, Rosario, "El uso del diminutivo en el lenguaje técnico latino", $R e$ vista de estudios latinos: RELat, 5, 2005, pp. 75-96.

LÓPEZ GREGORIS, Rosario, "El diminutivo latino: entre la denotación y la connotación", en Semántica latina y románica: unidades de significado conceptual y procedimental, Madrid, Universidad Autónoma de Madrid, 2016, pp. 177-198.

Marchese, Floriana, "Aspetti della lingua technica di Apicio", Atti e Memorie dell'Accademia Toscana La Colombaria, XXXVIII, 1987, pp. 9-102.

Milham, Mary Ella, "Aspects of Non-Technical Vocabulary in Apicius", The American Journal of Philology, 80/1, 1959, pp. 67-75.

Montero CARTElle, Enrique, "La literatura técnica latina de época tardía: aspectos lingüísticos y literarios", Cuadernos de literatura griega y latina, IV, 2003, pp. 259-280.

Norrick, Neal R., "Recipes as texts: technical language in the kitchen", en René Jongen, Sabine De Knop, Peter H. Nelde and Marie-Paul Quix (eds.), Sprache, Diskurs und Text. Akten des 17 Linguistischen Kolloquiums, Brüsel, 1982, Tübingen, Max Niemeyer Verlag, 1983, pp. 173-181.

Pickstone, Joan E., "Roman cookery”, Greece and Rome, IV, 1935, pp. 168-174.

Real Academia de la Lengua, Diccionario de la lengua española, edición del Tricentenario, actualización 2018, https://dle.rae.es/?w=diccionario (17/07/2020).

Tame de Patjane, Ma. Ivonne, Único diccionario internacional de cocina, México, s. e., 1998.

VÄÄNÄNEN, Veikko, Introducción al latín vulgar, trad. española Manuel Carrión, Madrid, Gredos, 1995.

Amalia Lejavitzer Lapoujade es doctora en Letras por la Universidad Nacional Autónoma de México (UNAM), miembro del Sistema Nacional de Investigadores de la Agencia Nacional de Investigación e Innovación del Uruguay, profesora de alta dedicación en el Departamento de Comunicación de la Universidad Católica del Uruguay (UCU). Ha sido profesora de la Facultad de Filosofía y Letras e investigadora de tiempo completo en el Centro de Estudios Clásicos del Instituto de Investigaciones Filológicas, UNAM. Tiene publicados diversos artículos especializados y de divulgación sobre historia de la alimentación y patrimonio cultural alimentario, así como una traducción del latín al español de los libros XIII y XIV de los Epigramas de Marcial (Hacia una génesis del epigrama en Marcial: Xenia y Apophoreta, México, unAm, 2000); su libro más reciente es Alimentación, salud y cultura en De re coquinaria de Apicio (México, unAm, 2018). Actualmente coordina el proyecto Patrimonio Cultural UCU y la línea de investigación Patrimonio cultural e identidad: ciudad, imagen y alimentación. Es estudiosa y traductora del De re coquinaria, especialista en alimentación en la antigua Roma y en Patrimonio cultural alimentario. 